development of the first UK wide palliative trainee research collaborative.

Methods The UK Palliative Care Trainee Research Collaborative (UKPRC) was established in 2017. Modelled on other successful trainee collaboratives, we employed the following steps:

1. Writing and adopting a constitution that enshrines shared authorship as a central tenant;

2. Identifying a core group of interested trainees;

3. Nominating a clinical and research lead;

4. Defining a brand; logo, website, email address, newsletter and twitter profile;

5. Choosing a project;

6. Recognising crucial stakeholders to disseminate information and garner support.

Results Launched publicly in August 2018, the UKPRC already has members from $12 / 14$ of the UK deaneries. The first national audit project is underway aiming to recruit 40 sites across all sectors in the UK. The UKPRC has a website, 186 followers on twitter and has been discussed by the APM and the National Clinical Research network leads in palliative and supportive care in the UK.

Conclusions The UKPRC has the potential to conduct audit and research across hospice and hospital sectors UK wide, which will inform evidence-based practice and ultimately aims to improve patient care. Collaboratives may strengthen the research culture within palliative medicine, embedding research activity and evidence-based practice from the first year of training.

\section{IMPACT OF AN EDUCATION PROGRAMME TO IMPROVE PRESCRIBING OF ANTICIPATORY MEDICATIONS IN THE COMMUNITY}

Rebecca Theobald, Julie Johns, Gail White, Sophie Northway. St Benedict's Hospice and Centre for Specialist Palliative Care, part of South Tyneside NHS Foundation Trust

10.1136/bmjspcare-2019-ASP.42

Background A baseline audit demonstrated a need to improve anticipatory prescribing at the end of life in a community setting. The specialist palliative care team facilitated education on end of life care prescribing. The impact of the education delivered was evaluated using a combination of methods.

Method Six sessions on anticipatory prescribing were delivered to 93 General Practitioners (GPs) in year one and five sessions to 56 GPs in year two. A mixture of teaching methods was used both years. Delegate feedback was collected for all sessions. Informal education/coaching with GPs was also provided. A prospective re-audit was conducted of 50 community prescription charts to identify any change in prescribing practice.

Results A re-audit showed anticipatory medication was prescribed more frequently for the five core symptoms. The increase in prescribing frequency ranged from 19\% to $49 \%$. This finding is consistent with an improvement in delegate confidence scores. However, half the patients had an error on their community prescription chart compared to $41 \%$ in the baseline audit. This should be considered in the context of each chart containing more prescriptions than in the previous audit. $26 \%$ had an error due to an incorrect frequency of as required medication. Other errors identified included the dose being too high $(8 \%)$ and too low (6\%).

Conclusion The audit showed a significant improvement in practice; with anticipatory medication for all five core symptoms prescribed more frequently. GPs need to prescribe anticipatory medication relatively infrequently which may contribute to the errors observed on half of the community prescription charts. To reduce such errors a commitment to on going education is required. Overall the education programme delivered by specialists in palliative care resulted in a positive change in practice.

\section{REAL TALK - BEYOND ADVANCED COMMUNICATION SKILLS: OUTCOMES OF A RESIDENTIAL WORKSHOP FOR PALLIATIVE CARE DOCTORS}

${ }^{1}$ Becky Whittaker, ${ }^{2}$ Sharan Watson, ${ }^{1}$ Marco Pino. 'Loughborough University; ${ }^{2}$ Derby University

10.1136/bmjspcare-2019-ASP.43

Background Analysis of filmed data of patient consultations at a UK hospice provides the materials for 'Real Talk'; a novel and flexible education intervention containing reallife film clips. Communication skills training is more likely to be effective in changing behaviours when it is experiential and interactive, being relevant to trainees' practice.

Methods Experienced palliative care doctors attended a threeday residential workshop in which they explored the Real Talk intervention in facilitated small groups. Discussions linked to the evidence relating to communication strategies, whilst reflective diaries and action planning provided opportunity for linking learning to their clinical and educator roles.

Results The workshop was attended by 29 experienced palliative care doctors who completed a pre and post questionnaire we adapted from a validated tool. Pre-workshop questions asked for workshop expectations; 19 delegates identified all their expectations had been met, 10 did not indicate an answer. Narratives from the expanded answers noted the workshop had exceeded expectations and the 'train the trainer' approach was welcomed. Delegates identified the most effective aspects of learning included experiential small group work relating to the content of the Real Talk film clips, opportunity to critique underpinning evidence of how clinicians communicate in relation to conversations in end of life care and having an opportunity to reflect on learning and application to practice in a safe and stimulating environment.

Conclusions Engagement in, and feedback on, the workshop has provided a foundation on which to build our research in understanding complex communication and skills training. Providing interactive experiential learning, embedded in the emerging evidence base underpinning Real Talk, is crucial for clinicians seeking to explore complex communication skills with patients facing the end of life. Ensuring skilled facilitation, a safe environment and programme flexibility are crucial to the learning process. 